\title{
CHARACTERIZING ATTAINABLE CLAIMS: A NEW PROOF
}

\author{
MICHAEL R. TEHRANCHI, ${ }^{*}$ University of Cambridge
}

\begin{abstract}
This short note offers a new proof of the following fact: in a discrete-time arbitrage-free market model, a contingent claim is attainable if and only if its expected value is the same under all equivalent martingale measures. The proof is based on Rogers' (1994) proof of the Dalang-Morton-Willinger (1990) theorem.
\end{abstract}

Keywords: Fundamental theorem of asset pricing; attainable contingent claim 2010 Mathematics Subject Classification: Primary 60G42; 91B25

\section{Introduction}

The purpose of this note is to offer a new proof of the well-known fact that a contingent claim can be replicated by a self-financing trading strategy if and only if it has a unique arbitrage-free price. To formulate this statement mathematically, let $S=\left(S_{t}\right)_{0 \leq t \leq T}$ be a fixed $d$-dimensional discrete-time stochastic process defined on a probability space $(\Omega, \mathcal{F}, \mathrm{P})$ and adapted to a filtration $\left(\mathcal{F}_{t}\right)_{0 \leq t \leq T}$, where we assume for simplicity that $\mathcal{F}_{0}$ is trivial and $\mathcal{F}=\mathcal{F}_{T}$. Let

$$
\mathcal{Q}=\{\mathrm{Q} \sim \mathrm{P}: S \text { is a } \mathrm{Q} \text {-martingale }\}
$$

be the set of equivalent martingale measures. For a $d$-dimensional predictable process $H=$ $\left(H_{t}\right)_{1 \leq t \leq T}$, let

$$
(H \cdot S)_{t}=\sum_{s=1}^{t} H_{s} \cdot \Delta S_{S}
$$

denote the discrete-time stochastic integral, where $a \cdot b$ denotes the standard inner product of vectors $a, b \in \mathbb{R}^{d}$ and $\Delta S_{t}=S_{t}-S_{t-1}$. Then the well-known fact quoted above amounts to the following theorem.

Theorem 1.1. Assume that $\mathcal{Q}$ is not empty. For a real-valued random variable $X$, the following statements are equivalent:

(a) $X=(H \cdot S)_{T}$ almost surely (a.s.) for some predictable process $H$;

(b) $\mathrm{E}^{\mathrm{Q}}(X)=0$ for all $\mathrm{Q} \in \mathcal{Q}$ such that $\mathrm{E}^{\mathrm{Q}}(|X|)<\infty$.

Theorem 1.1 has been in the folklore of financial mathematics for at least a generation. For instance, Ross [11] in his 1978 paper wrote ' ... if all admissible operators [i.e. expectations with respect to equivalent martingale measures] lead to the same value, then the return stream must be spanned by existing assets and the resulting value is the proper one for the investment

Received 30 April 2010; revision received 28 July 2010.

* Postal address: Statistical Laboratory, University of Cambridge, Centre for Mathematical Sciences, Wilberforce Road, Cambridge CB3 0WB, UK. Email address: m.tehranchi@statslab.cam.ac.uk 
criterion.' Indeed, Theorem 1.1 is announced as Corollary (b) of Theorem 2 of Harrison and Kreps's [4] 1979 paper.

To illustrate the issues involved, let us consider the case when $T=1$ and the sample space $\Omega$ has $n$ elements. Without loss of generality, we will assume that $\mathrm{P}\{\omega\}>0$ for each $\omega \in \Omega$. To switch to linear-algebraic notation, we define a $d \times n$ matrix $A$ by

$$
A_{i, j}=\Delta S_{1}^{i}\left(\omega_{j}\right)
$$

The set

$$
\hat{\mathcal{Q}}=\left\{q \in \mathbb{R}^{n}: A q=0, q_{j}>0 \text { for all } j, \text { and } q_{1}+\cdots+q_{n}=1\right\}
$$

corresponds to the set of equivalent martingale measures $\mathcal{Q}$ by the identification $\mathrm{Q}\left\{\omega_{j}\right\}=q_{j}$. A scalar random variable $X$ corresponds to a vector $x \in \mathbb{R}^{n}$, so that in this notation Theorem 1.1 says the following statements are equivalent:

(a) $x=A^{\top} h$ for some $h \in \mathbb{R}^{d}$;

(b) $q \cdot x=0$ for all $q \in \hat{\mathcal{Q}}$.

The direction (a) $\Rightarrow$ (b) is easy: if $x=A^{\top} h$ for some $h \in \mathbb{R}^{d}$ then $q \cdot x=(A q) \cdot h=0$ for all $q \in \hat{Q}$. We now consider the direction (b) $\Rightarrow$ (a). By the orthogonal decomposition $\mathbb{R}^{n}=\operatorname{Ran} A^{\top} \oplus \operatorname{Ker} A$, it is enough to show that if $A x=0$ and $q \cdot x=0$ for all $q \in \hat{Q}$, then $x=0$.

Fix an element $q_{0} \in \hat{\mathcal{Q}}$. There exists a $\varepsilon \neq 0$ such that

$$
q_{\varepsilon}=\frac{\varepsilon x+q_{0}}{\varepsilon\left(x_{1}+\cdots+x_{n}\right)+1}
$$

is an element of $\hat{\mathcal{Q}}$. Indeed, if $\varepsilon$ is small enough then

$$
\left(\varepsilon x+q_{0}\right)_{j}>0 \text { for all } j .
$$

Since $q \cdot x=0$ for all $q \in \hat{\mathcal{Q}}$, we can conclude that

$$
0=q_{\varepsilon} \cdot x=|x|^{2} \frac{\varepsilon}{\varepsilon\left(x_{1}+\cdots+x_{n}\right)+1},
$$

and, hence, $x=0$ as claimed.

When the sample space $\Omega$ is infinite, a naive attempt to extend the above proof fails, even when $T=1$. Suppose for simplicity that $\Delta S_{1} \in L^{2}$, and let $X \in L^{2}$ be such that $\mathrm{E}\left(X \Delta S_{1}\right)=0$ and $\mathrm{E}^{\mathrm{Q}}(X)=0$ for all $\mathrm{Q} \in \mathcal{Q}$ for which $X$ in integrable. Fix one such measure $\mathrm{Q}_{0}$ with density

$$
Z_{0}=\frac{\mathrm{dQ}_{0}}{\mathrm{dP}}
$$

As before, we can let

$$
Z_{\varepsilon}=\frac{\varepsilon X+Z_{0}}{\varepsilon \mathrm{E}(X)+1} .
$$

However, we cannot proceed because there is no guarantee that we can choose a $\varepsilon \neq 0$ small enough such that $Z_{\varepsilon}>0$ a.s. Indeed, it may well be the case that ess $\inf Z_{0}=0$, ess $\inf X=$ $-\infty$, and ess $\sup X=\infty$.

The problem with the above approach is that the set $\left\{Z \in L^{2}: Z>0\right\}$ is generally not open. Indeed, a functional analytic proof of Theorem 1.1, even in the $T=1$ case, requires a 
more careful choice of topologies. The aim of this note is to offer a proof of Theorem 1.1 that by-passes these considerations. Aside from Jensen's inequality, the new proof of this theorem relies on the following representation results for elements of $\mathcal{Q}$.

Theorem 1.2. The following statements are equivalent:

(a) $(H \cdot S)_{T}=0$ a.s. for every predictable $H$ such that $(H \cdot S)_{T} \geq 0$ a.s.;

(b) the set $\mathbb{Q}$ is not empty;

(c) for every random variable $X$, there exist a positive random variable $Z$, predictable processes $H_{0}$ and $H_{1}$, positive constants $C_{0}$ and $C_{1}$, and (not necessarily distinct) probability measures $\mathrm{Q}_{0}$ and $\mathrm{Q}_{1}$ in $\mathcal{Q}$ such that

$$
\frac{\mathrm{dQ}_{0}}{\mathrm{dP}}=C_{0} Z \mathrm{e}^{\left(H_{0} \cdot S\right)_{T}} \quad \text { and } \quad \frac{\mathrm{dQ}_{1}}{\mathrm{dP}}=C_{1} Z \mathrm{e}^{\left(H_{1} \cdot S\right)_{T}+X} .
$$

Furthermore, $Z$ can be chosen such that the random variable

$$
\max \left\{1,|X|,\left|\left(H_{0} \cdot S\right)_{T}\right|,\left|\left(H_{1} \cdot S\right)_{T}\right|\right\} \max \left\{\frac{\mathrm{dQ}_{0}}{\mathrm{dP}}, \frac{\mathrm{dQ}_{1}}{\mathrm{dP}}\right\}
$$

is bounded.

The above theorem is a version of the fundamental theorem of asset pricing. Note that statement (a) above says that the market modelled by $S$ is free of arbitrage opportunities, since any trading strategy $H$ whose return $(H \cdot S)_{T}$ is always nonnegative, must, in fact, always return exactly 0 . On the other hand, condition (b) says that there exists at least one equivalent martingale measure. What is significant for us is that condition (b) implies condition (c): that the existence of one equivalent martingale measure automatically implies the existence of two martingale measures with densities given by explicit formulae. The idea of the new proof of Theorem 1.1 is to exploit these formulae when $\mathrm{E}^{\mathrm{Q}}(X)=0$ for $\mathrm{Q}=\mathrm{Q}_{0}$ and $\mathrm{Q}_{1}$ to find a representation of $X$ of the form $X=(H \cdot S)_{T}$. In fact, we can weaken condition (b) of Theorem 1.1 to

(b $\mathrm{b}^{\prime} \mathrm{E}^{\mathrm{Q}}(X)=0$ for all $\mathrm{Q} \in \mathcal{Q}$ such that $\max \{1,|X|\} \mathrm{dQ} / \mathrm{dP}$ is bounded,

since the measures $\mathrm{Q}_{0}$ and $\mathrm{Q}_{1}$ verify this boundedness property.

The equivalence (a) $\Leftrightarrow$ (b) in Theorem 1.2 is due to Dalang et al. [1], though Harrison and Pliska [5] proved the theorem for the case of a finite sample space $\Omega$. The above formulation of the fundamental theorem of asset pricing is implicit in the papers of Rogers [9], [10]. His proof in the case when $T=1$, reproduced in Section 3, is simple enough to be included in an advanced undergraduate course on financial mathematics. Indeed, the proof uses only the Bolzano-Weierstrass theorem and the fact that the gradient of a differentiable function vanishes at its maximum. The same proof can be made to work for $T>1$, but one must take care with the measure-theoretic technicalities.

There are also versions of Theorems 1.1 and 1.2 in continuous time. There is a significant difference between the discrete- and continuous-time theories because we have to be very careful in how to define a class of predictable processes $H$ such that the integral $(H \cdot S)$ is economically meaningful. Indeed, Harrison and Kreps [4] in 1979 already noticed that so-called doubling strategies must not be admissible, otherwise the notion of arbitrage becomes vacuous. In this setting, Jacka [6] proved the appropriate continuous-time version of Theorem 1.1, again 
confirming the folklore that a claim is attainable if and only if it has the expectation under all equivalent martingale measures. As for the continuous-time version of the fundamental theorem of asset pricing, Delbaen and Schachermayer [2] proved that a market model has no free lunch with vanishing risk if and only if there exists a probability measure under which the asset prices are $\sigma$-martingales. See the recent book of Delbaen and Schachermayer [3] for a survey of these results. Unfortunately, the method of proof detailed below for the discrete-time case does not seem to be directly applicable to the continuous-time case.

The note is arranged as follows. In Section 2, a proof of Theorem 1.1 is presented, including the main contribution of this note-a new, short proof of the implication (b) $\Rightarrow$ (a). In Section 3 we provide the details of Rogers's proof of Theorem 1.2 to keep the note self-contained.

\section{A proof of Theorem 1.1}

Proof of Theorem 1.1. $(a) \Rightarrow(b)$. Let $H$ be predictable and define a process $Y$ by $Y_{t}=$ $(H \cdot S)_{t}$. Suppose that the random variable $Y_{T}$ is integrable with respect to some fixed Q for which $S$ is a martingale. The case $T=1$ proceeds exactly as the finite $\Omega$ case discussed in the introduction: $\mathrm{E}\left(Y_{1}\right)=H_{1} \cdot \mathrm{E}\left(\Delta S_{1}\right)=0$ since $H_{1}$ is not random. However, the case $T>1$ takes a modicum of care. It is sufficient to show that $Y$ is a martingale since $Y_{0}=0$.

We would like to show that if $Y_{t}$ is integrable for some $0<t \leq T$ then $\mathrm{E}\left(Y_{t} \mid \mathcal{F}_{t-1}\right)=Y_{t-1}$. This would imply that $Y_{t-1}$ is integrable because $\mathrm{E}\left(\left|Y_{t-1}\right|\right) \leq \mathrm{E}\left(\left|Y_{t}\right|\right)<\infty$. Since $Y_{T}$ is integrable by assumption, this would show that $Y$ is a martingale by (backward) induction.

Fix $t$, suppose that $Y_{t}$ is integrable, and define an increasing sequence of $\mathcal{F}_{t-1}$-measurable events

$$
A_{n}=\left\{\left|Y_{t-1}\right| \leq n,\left|H_{t}\right| \leq n\right\}
$$

on which both $Y_{t-1}$ and $H_{t}$ are bounded. Note the equality

$$
\begin{aligned}
\mathrm{E}\left(\mathbf{1}_{A_{n}} Y_{t} \mid \mathcal{F}_{t-1}\right) & =\mathrm{E}\left(\mathbf{1}_{A_{n}} Y_{t-1} \mid \mathcal{F}_{t-1}\right)+\mathrm{E}\left(\mathbf{1}_{A_{n}} H_{t} \cdot \Delta S_{t} \mid \mathcal{F}_{t-1}\right) \\
& =\mathbf{1}_{A_{n}} Y_{t-1}+\mathbf{1}_{A_{n}} H_{t} \cdot \mathrm{E}\left(\Delta S_{t} \mid \mathcal{F}_{t-1}\right) \\
& =\mathbf{1}_{A_{n}} Y_{t-1} .
\end{aligned}
$$

Since $Y_{t}$ is assumed integrable, we can apply the conditional dominated convergence theorem to send $n \rightarrow \infty$ to get $\mathrm{E}\left(Y_{t} \mid \mathcal{F}_{t-1}\right)=Y_{t-1}$ as desired.

$(b) \Rightarrow(a)$. This is the main contribution of this note. Assume that $\mathcal{Q}$ is not empty, and that $\mathrm{E}^{\mathrm{Q}}(X)=0$ for all $\mathrm{Q} \in \mathcal{Q}$ for which $X$ is integrable. Applying Theorem 1.2, we note that

$$
X=x+(H \cdot S)_{T}+\log \left(\frac{\mathrm{dQ}_{1}}{\mathrm{dQ}_{0}}\right)
$$

for a predictable process $H=H_{0}-H_{1}$, probability measures $\mathrm{Q}_{0}, \mathrm{Q}_{1} \in \mathcal{Q}$, and a constant $x=\log \left(C_{0} / C_{1}\right)$. We need to show that $x=0$ and $\mathrm{Q}_{0}=\mathrm{Q}_{1}$. Again, by Theorem 1.2 we know that $X$ and $(H \cdot S)_{T}$ are integrable with respect to both $\mathrm{Q}_{0}$ and $\mathrm{Q}_{1}$. Now by assumption we have $\mathrm{E}^{\mathrm{Q}}(X)=0$, and by the implication $(\mathrm{a}) \Rightarrow(\mathrm{b})$ of this theorem, proven above, we have $\mathrm{E}^{\mathrm{Q}}\left[(H \cdot S)_{T}\right]=0$ for both $\mathrm{Q}=\mathrm{Q}_{0}$ and $\mathrm{Q}_{1}$. 
Integrating (2.1) and using Jensen's inequality yields

$$
\begin{aligned}
x & =-\mathrm{E}^{\mathrm{Q}_{0}} \log \left(\frac{\mathrm{dQ}_{1}}{\mathrm{dQ}_{0}}\right) \\
& \geq-\log \mathrm{E}^{\mathrm{Q}_{0}}\left(\frac{\mathrm{dQ}_{1}}{\mathrm{dQ}_{0}}\right) \\
& =0 \\
& =\log \mathrm{E}^{\mathrm{Q}_{1}}\left(\frac{\mathrm{dQ}_{0}}{\mathrm{dQ}_{1}}\right) \\
& \geq \mathrm{E}^{\mathrm{Q}_{1}} \log \left(\frac{\mathrm{dQ}_{0}}{\mathrm{dQ}_{1}}\right) \\
& =x,
\end{aligned}
$$

so that $x=0$. But since the logarithmic function is strictly concave, Jensen's inequality holds with equality only if $d Q_{1} / d Q_{0}$ is constant. Since $Q_{0}$ and $Q_{1}$ are probability measures, we must conclude that $\mathrm{Q}_{0}=\mathrm{Q}_{1}$, as desired.

\section{Rogers' proof of the fundamental theorem of asset pricing}

For completeness, we give a proof of Theorem 1.2. We do this in stages.

Proof of Theorem 1.2. $(b) \Rightarrow(a)$. Suppose that $\mathcal{Q}$ is not empty, and that $(H \cdot S)_{T} \geq 0$ a.s. Let $Y=(H \cdot S)$, and fix $\mathrm{Q} \in \mathcal{Q}$.

To see the structure of the argument, we first consider the case in which $T=1$. Since $H_{1}$ constant, the random variable $Y_{1}=H_{1} \cdot \Delta S_{1}$ is integrable. Since $Y_{1} \geq 0$ a.s. but $\mathrm{E}\left(Y_{1}\right)=$ $H_{1} \cdot \mathrm{E}\left(\Delta S_{1}\right)=0$, we must conclude that $Y_{1}=0$ a.s.

Now we consider the case in which $T>1$. We will first show that $Y_{t} \geq 0$ a.s. for all $0 \leq t \leq T$. We proceed by induction. Suppose that $Y_{t} \geq 0$ a.s. for some fixed $0<t \leq T$, and let

$$
A_{n}=\left\{\left|Y_{t-1}\right| \leq n,\left|H_{t}\right| \leq n\right\} .
$$

As before, we have

$$
0 \leq \mathrm{E}\left(\mathbf{1}_{A_{n}} Y_{t} \mid \mathcal{F}_{t-1}\right)=\mathbf{1}_{A_{n}} Y_{t-1} \rightarrow Y_{t-1}
$$

as $n \rightarrow \infty$, so that $Y_{t-1} \geq 0$ a.s. Since $Y_{T} \geq 0$ a.s. by assumption, we can conclude that $Y_{t} \geq 0$ a.s. for all $0 \leq t \leq T$ by induction.

We now show that $Y_{t}=0$ for all $0 \leq t \leq T$. Now suppose that $Y_{t-1}=0$ a.s. for some fixed $0<t \leq T$. Then

$$
0=\mathbf{1}_{A_{n}} Y_{t-1}=\mathrm{E}\left(\mathbf{1}_{A_{n}} Y_{t} \mid \mathcal{F}_{t-1}\right)
$$

for every $n$. But since $\mathbf{1}_{A_{n}} Y_{t} \geq 0$ a.s., we must conclude that $\mathbf{1}_{A_{n}} Y_{t}=0$ a.s. Letting $n \rightarrow \infty$ then implies that $Y_{t}=0$ a.s. Since $Y_{0}=0$, the (forward) induction is complete.

Remark 3.1. It should be noted that neither the proof of the $(a) \Rightarrow$ (b) direction of Theorem 1.1 nor the proof of the (b) $\Rightarrow$ (a) direction of Theorem 1.2 given above are especially new and are included only for completeness. Similar ideas can be found, for instance, in Jacod and Shiryaev's [7] proof of the fundamental theorem of asset pricing.

Since the $(c) \Rightarrow$ (b) implication of Theorem 1.2 is self-evident, it only remains to prove the (a) $\Rightarrow$ (c) direction. 
Remark 3.2. We now digress from the formal proof to outline the motivation behind the manipulations that follow. Rogers's proof consists of two steps. The first step is to look at the classical problem of maximizing the expected utility of terminal wealth. Indeed, let $U$ be a smooth, strictly increasing, and concave utility function on $\mathbb{R}$ modelling an investor's aversion to risk, and consider the problem

\section{maximize $\mathrm{E} U\left[(H \cdot S)_{T}\right]$}

over predictable trading strategies $H$. If there exists a maximizer $H^{*}$ then the following idea is well known to economists: the marginal utility $U^{\prime}\left[\left(H^{*} \cdot S\right)_{T}\right]$ of the optimal terminal wealth is proportional to the density of an equivalent martingale measure. To see why, let us suppose for the moment that the sample space $\Omega$ is finite to avoid discussing technicalities, although it should be stressed that $\Omega$ may be infinite in the proof that follows. Now, for any trading strategy $\eta$, the function

$$
\varepsilon \mapsto \operatorname{E} U\left[\left(H^{*} \cdot S\right)_{T}+\varepsilon(\eta \cdot S)_{T}\right]
$$

is maximized at $\varepsilon=0$, so that

$$
\left.\frac{\mathrm{d}}{\mathrm{d} \varepsilon} \mathrm{E} U\left[\left(H^{*} \cdot S\right)_{T}+\varepsilon(\eta \cdot S)_{T}\right]\right|_{\varepsilon=0}=\mathrm{E}\left(U^{\prime}\left[\left(H^{*} \cdot S\right)_{T}\right](\eta \cdot S)_{T}\right)=0
$$

by Fermat's first-order condition for a maximum. Since $\eta$ was arbitrary, we immediately see that the probability measure $\mathrm{Q}^{*}$ with density

$$
\frac{\mathrm{dQ}^{*}}{\mathrm{dP}}=\frac{U^{\prime}\left[\left(H^{*} \cdot S\right)_{T}\right]}{\mathrm{E} U^{\prime}\left[\left(H^{*} \cdot S\right)_{T}\right]}
$$

is an equivalent martingale measure.

By modifying the above argument we can find other formulae for densities of elements of $\mathcal{Q}$. Indeed, given random variables $X$ and $Z$, where $Z>0$ a.s., consider the new problem

$$
\operatorname{maximize} \mathrm{E}\left(Z U\left[(H \cdot S)_{T}+X\right]\right) \text {. }
$$

As before, if there exists a maximizer $H^{\star}$ to the problem, then the first-order condition yields an equivalent martingale measure $\mathrm{Q}^{\star}$ with density

$$
\frac{\mathrm{dQ}}{\mathrm{dP}}=C Z U^{\prime}\left[\left(H^{\star} \cdot S\right)_{T}+r X\right]
$$

for a normalizing constant $C>0$. It is this modified formulation of the utility maximization idea that will give us the formulae given by condition (c), where we will take, modulo some changes of signs, $U(x)=-\mathrm{e}^{-x}$.

The second step of Rogers's proof of Theorem 1.2 consists of showing the no-arbitrage condition implies that a well-chosen utility maximization problem always has a solution.

Proof of Theorem 1.2. $(a) \Rightarrow(c)$. To see the idea of the proof, we first consider the case in which $T=1$. Let $Z=\exp \left(-X^{2}-\left|\Delta S_{1}\right|^{2}\right)$, and consider two functions $F_{0}$ and $F_{1}$ on $\mathbb{R}^{d}$ defined by

$$
F_{r}(h)=\mathrm{E}\left[Z \exp \left(h \cdot \Delta S_{1}+r X\right)\right] .
$$

Note that each $F_{r}$ is nothing but the moment generating function of $\Delta S_{1}$ with respect to the measure whose density with respect to $\mathrm{P}$ is $Z \mathrm{e}^{r X}$. Each $F_{r}$ is everywhere finite valued, thanks to the choice of $Z$, and, hence, smooth, and, in particular,

$$
\nabla F_{r}(h)=\mathrm{E}\left[Z \exp \left(h \cdot \Delta S_{1}+r X\right) \Delta S_{1}\right] .
$$


Now, if we can show that there exist vectors $h_{r} \in \mathbb{R}^{d}$ which minimize $F_{r}$ then we are done. Indeed, we would have

$$
0=\nabla F_{r}\left(h_{r}\right)=\mathrm{E}\left[Z \exp \left(h_{r} \cdot \Delta S_{1}+r X\right) \Delta S_{1}\right]
$$

by the first-order condition for the minimizer of a smooth function, and, hence, the densities

$$
\frac{\mathrm{dQ} r}{\mathrm{dP}}=C_{r} Z \exp \left(h_{r} \cdot \Delta S_{1}+r X\right)
$$

define equivalent martingale measures, where the $C_{r}>0$ are normalizing constants. Note that, by the choice of $Z$, the random variable

$$
\max \left\{1, X,\left|h_{0} \cdot \Delta S_{1}\right|,\left|h_{1} \cdot \Delta S_{1}\right|\right\} \max \left\{\frac{\mathrm{dQ}_{0}}{\mathrm{dP}}, \frac{\mathrm{dQ}_{1}}{\mathrm{dP}}\right\}
$$

is bounded.

We must now show that the minimizers indeed exist. Fix an $r \in\{0,1\}$, and drop it from the notation. Now let $\left(h_{n}\right)_{n}$ be a minimizing sequence, so that

$$
F\left(h_{n}\right) \rightarrow \inf _{h} F(h) .
$$

If $\left(h_{n}\right)_{n}$ is bounded then there exists a convergent subsequence, still denoted $\left(h_{n}\right)_{n}$ so that $h_{n} \rightarrow h^{*}$. But since $F$ is smooth, we have $F\left(h_{n}\right) \rightarrow F\left(h^{*}\right)=\inf _{h} F(h)$, and, hence, $h^{*}$ is our desired minimizer.

It remains to rule out the possibility that $\left(h_{n}\right)_{n}$ is unbounded. So we now suppose that $\left(h_{n}\right)_{n}$ is unbounded and aim to show that there exists an arbitrage. Let $\mathcal{V}$ be smallest subspace of $\mathbb{R}^{d}$ containing the support of the random vector $\Delta S_{1}$. That is, $\mathcal{V}$ is the orthogonal complement of the subspace

$$
u=\left\{u \in \mathbb{R}^{d}: u \cdot \Delta S_{1}=0 \text { a.s. }\right\} .
$$

In particular, we will use the fact that if $v \in \mathcal{V}$ and $v \neq 0$, then $\mathrm{P}\left\{v \cdot \Delta S_{1}=0\right\}<1$.

Note that if $u \in \mathcal{U}$ and $v \in \mathcal{V}$, then $F(u+v)=F(v)$. Hence, we may suppose that the minimizing sequence is such that $h_{n} \in \mathcal{V}$ for all $n$ and $\left|h_{n}\right| \rightarrow \infty$.

Letting $\hat{h}_{n}=h_{n} /\left|h_{n}\right|$ and noting that the sequence $\left(\hat{h}_{n}\right)_{n}$ is bounded, we can replace our sequence $\left(h_{n}\right)_{n}$ with a subsequence such that $\hat{h}_{n}$ converges to a unit vector $\hat{h} \in \mathcal{V}$. Clearly,

$$
\exp \left(h_{n} \cdot \Delta S_{1}\right)=\left(\exp \left(\hat{h}_{n} \cdot \Delta S_{1}\right)\right)^{\left|h_{n}\right|} \rightarrow \infty
$$

on the event $\left\{\hat{h} \cdot \Delta S_{1}>0\right\}$, but since we have

$$
\begin{aligned}
\mathrm{E}\left[\liminf _{n} Z \exp \left(h_{n} \cdot \Delta S_{1}+r X\right)\right] & \leq \liminf _{n} \mathrm{E}\left[Z \exp \left(h_{n} \cdot \Delta S_{1}+r X\right)\right] \\
& =\inf _{h} F(h) \\
& \leq F(0) \\
& <\infty
\end{aligned}
$$

by Fatou's lemma, we must conclude that $\mathrm{P}\left\{\hat{h} \cdot \Delta S_{1}>0\right\}=0$ so that $-\hat{h} \cdot \Delta S_{1} \geq 0$ a.s. But since $\hat{h} \in \mathcal{V}$ and $\hat{h} \neq 0$, we must have $\mathrm{P}\left\{\hat{h} \cdot \Delta S_{1}=0\right\}<1$. Hence, $-\hat{h}$ is an arbitrage, contradicting the no-arbitrage assumption (a), completing the proof in the $T=1$ case. 
Now, we consider the case in which $T>1$. The idea is the same as above, but we now have to worry about measurability and integrability. We will build up the processes $H_{r}$ inductively. Suppose that we have constructed the random vectors $H_{r}(t+1), \ldots, H_{r}(T)$ for both $r \in\{0,1\}$, where we begin the procedure at $t=T$ when nothing has been constructed. We now construct $H_{r}(t)$. As before, we introduce a random factor to ensure integrability. Let

$$
Z_{t}=\exp \left(-X^{2}-\sum_{1 \leq s \leq T}\left|\Delta S_{s}\right|^{2}-\sum_{t+1 \leq s \leq T}\left(\left|H_{0}(s)\right|^{2}+\left|H_{1}(s)\right|^{2}\right)\right) .
$$

Fix $t$ and $r$, and let $P$ be a regular conditional distribution of the random vector

$$
\left(\Delta S_{t}, X, \sum_{t+1 \leq s \leq T} H_{r}(s) \cdot \Delta S_{s}, Z_{t}\right)
$$

given $\mathcal{F}_{t}$. Now define a function $F: \mathbb{R}^{d} \times \Omega \rightarrow \mathbb{R}$ by

$$
\begin{aligned}
F(h, \omega) & =\int z \mathrm{e}^{r x+h \cdot w+y} \mathrm{~d} P(w, x, y, z ; \omega) \\
& =\mathrm{E}\left[Z_{t} \exp \left(r X+h \cdot \Delta S_{t}+\sum_{t+1 \leq s \leq T} H_{r}(s) \cdot \Delta S_{s}\right) \mid \mathcal{F}_{t-1}\right](\omega) .
\end{aligned}
$$

Note that, for each $\omega \in \Omega$, the function $F(\cdot, \omega)$ is smooth.

For each $\omega \in \Omega$, we will find an $H_{r}(t, \omega)$ which minimizes $F(\cdot, \omega)$. Let us assume for the moment that this minimizer exists and is $\mathcal{F}_{t-1}$-measurable. The first-order condition for a minimum applies and we have

$$
\nabla F\left(H_{r}(t)\right)=\mathrm{E}\left[Z_{t} \exp \left(r X+\sum_{t \leq s \leq T} H_{r}(s) \cdot \Delta S_{s}\right) \Delta S_{t} \mid \mathscr{F}_{t-1}\right]=0 .
$$

At the end of the induction, we will set

$$
\frac{\mathrm{dQ} r}{\mathrm{dP}}=C_{r} Z_{1} \exp \left(r X+\left(H_{r} \cdot S\right)_{T}\right)
$$

for an appropriate normalizing constant $C_{r}>0$. The above calculation shows that $\mathrm{Q}_{r}$ is indeed an equivalent martingale measure. By the choice of $Z=Z_{1}$, it satisfies the announced boundedness property too.

The remaining part of the proof is to confirm that an $\mathcal{F}_{t-1}$-measurable minimizer $H_{r}(t)$ exists. As before, we need to consider a minimizing sequence. However, since $\mathcal{F}_{t-1}$ is generally nontrivial, we need to take a little care with measurability. We consider a sequence of regularized objective functions

$$
F_{n}(h, \omega)=F(h, \omega)+\frac{1}{n}|h|^{2} .
$$

Note that, for all $\omega \in \Omega$, the function $F_{n}(\cdot, \omega)$ is strictly convex, and that $F_{n}(h, \omega) \rightarrow \infty$ as $|h| \rightarrow \infty$. Hence, there exists a unique minimizer $H_{n}(\omega)$. Since

$$
F\left(H_{n}\right) \leq F_{n}\left(H_{n}\right) \leq F_{n}(h)=F(h)+\frac{1}{n}|h|^{2} \text { for all } h \in \mathbb{R}^{d},
$$


we can first take the lim sup as $n \rightarrow \infty$ and then take the infimum over $h \in \mathbb{R}^{d}$ to conclude that $F\left(H_{n}(\omega), \omega\right) \rightarrow \inf _{h} F(h, \omega)$ for each $\omega$.

This minimizer $H_{n}$ is $\mathcal{F}_{t-1}$-measurable since

$$
\left\{H_{n} \in B\right\}=\bigcup_{p \in Q^{d}} \bigcap_{q \in Q^{d}}\left\{p \in B, q \in B^{\mathrm{c}}, F_{n}(p)<F_{n}(q)\right\}
$$

for all open balls $B \subset \mathbb{R}^{d}$, where $Q^{d}$ is the countable set of vectors in $\mathbb{R}^{d}$ with rational coordinates.

Let $A$ be the $\mathcal{F}_{t-1}$-measurable event

$$
A=\bigcup_{k} \bigcap_{n}\left\{\left|H_{n}\right|<k\right\}
$$

that the sequence $\left(H_{n}\right)_{n}$ is bounded. By Lemma 3.1 below, there exists an increasing sequence of measurable indices $N_{k}: A \rightarrow \mathbb{N}$ such that $H_{N_{k}}(\omega)$ converges to some $H(\omega) \in \mathbb{R}^{d}$ for each $\omega \in A$. Since $F_{N_{k}}\left(H_{N_{k}}\right) \rightarrow F(H)$ pointwise on $A$, we see that $H=H_{r}(t)$ will be our desired minimizer as long as we show that $\mathrm{P}\{A\}=1$.

Now, define the subspace $U$ orthogonal to the conditional support of $\Delta S_{t}$ given $\mathcal{F}_{t-1}$ by

$$
\mathcal{U}(\omega)=\left\{u \in \mathbb{R}^{d}: P\left(\left\{w \in \mathbb{R}^{d}: u \cdot w=0\right\}, \omega\right)=1\right\},
$$

and let $\mathcal{V}(\omega)=\mathcal{U}(\omega)^{\perp}$. We see that the minimizer $H_{n}(\omega)$ is in $\mathcal{V}(\omega)$ for each $\omega$ since

$$
F_{n}(u+v) \geq F_{n}(v)
$$

whenever $u \in \mathcal{U}$ and $v \in \mathcal{V}$.

Since $\left(H_{n}(\omega)\right)_{n}$ is unbounded for each $\omega \in A^{\mathrm{c}}$, we can find a measurable sequence of indices $N_{k}: A^{\mathrm{c}} \rightarrow \mathbb{N}$ such that $\left|H_{N_{k}}\right| \rightarrow \infty$ on $A^{\mathrm{c}}$, by taking $N_{k}=\inf \left\{n:\left|H_{n}\right|>k\right\}$. Furthermore, since the sequence $\hat{H}_{N_{k}}=H_{N_{k}} /\left|H_{N_{k}}\right|$ is bounded on $A^{\mathrm{c}}$, we can find another measurable sequence of indices, again by Lemma 3.1, which we continue to denote by $N_{k}$, such that $\hat{H}_{N_{k}}(\omega)$ converges to $\hat{H}(\omega)$ on $A^{\text {c }}$. Note that $\hat{H}(\omega)$ is a unit vector in $\mathcal{V}(\omega)$ for each $\omega \in A^{\mathrm{c}}$. We can extend $\hat{H}$ to an $\widetilde{F}_{t-1}$-measurable random vector by setting $\hat{H}=0$ on $A$. As before,

$$
\exp \left(H_{N_{k}} \cdot \Delta S_{t}\right)=\left(\exp \left(\hat{H}_{N_{k}} \cdot \Delta S_{t}\right)\right)^{\left|H_{N_{k}}\right|} \rightarrow \infty
$$

on the event $A^{\mathrm{c}} \cap\left\{\hat{H} \cdot \Delta S_{t}>0\right\}$. But by Fatou's lemma we have

$$
\begin{aligned}
\mathrm{E}\left(\liminf _{k \rightarrow \infty} \exp \left(H_{N_{k}} \cdot \Delta S_{t}\right) \eta \mid \mathcal{F}_{t-1}\right)(\omega) & \leq \liminf _{k \rightarrow \infty} F\left(H_{N_{k}}(\omega), \omega\right) \\
& =\inf _{h} F(h, \omega) \leq F(0, \omega) \\
& <\infty
\end{aligned}
$$

for all $\omega \in A^{\mathrm{c}}$, where $\eta=Z_{t} \exp \left(r X+\sum_{t+1 \leq s \leq T} H_{r}(s) \cdot \Delta S_{s}\right)$. Hence $\mathrm{P}\left\{A^{c} \cap\left\{\hat{H} \cdot \Delta S_{t}>\right.\right.$ $0\}\}=0$. But this implies that $-\mathbf{1}_{A^{\mathrm{c}}} \hat{H} \cdot \Delta S_{t} \geq 0$ a.s. Now, using the no-arbitrage condition (a), we can conclude that $\mathbf{1}_{A^{\mathrm{c}}} \hat{H} \cdot \Delta S_{t}=0$ a.s. But this means that $\mathbf{1}_{A^{\mathrm{c}}} \hat{H} \in \mathcal{U}$. But since $\hat{H} \in \mathcal{V}=U^{\perp}$, we must conclude that $\mathrm{P}\left\{A^{\mathrm{c}}\right\}=0$, as desired.

We now state and prove the technical lemma used in the proof above. It is, in a sense, a measurable version of the Bolzano-Weierstrass theorem. It appears in the paper of Kabanov and Stricker [8], who thanked Engelbert and Weizsäcker for suggesting it. 
Lemma 3.1. Consider a sequence of measurable functions $\xi_{n}: B \rightarrow \mathbb{R}^{d}$ such that

$$
\sup _{n}\left|\xi_{n}(\omega)\right|<\infty \quad \text { for all } \omega \in B
$$

Then there exists an increasing sequence of measurable indices $N_{k}: B \rightarrow \mathbb{N}$ such that $\left(\xi_{N_{k}}(\omega)\right)_{k}$ converges for each $\omega \in B$.

Proof. We consider the case $d=1$ first. Let

$$
N_{k}=\inf \left\{h \geq 1: \xi_{h} \geq \limsup _{n \rightarrow \infty} \xi_{n}-\frac{1}{k}\right\} .
$$

It is easy to see that the $N_{k}$ are measurable, so that the $\xi_{N_{k}}$ are also measurable and $\xi_{N_{k}} \rightarrow$ $\lim \sup _{n \rightarrow \infty} \xi_{n}$ pointwise.

For the case $d>1$, we simply work componentwise. First let $N_{k}^{0}=k$ for all $k \geq 1$, and define recursively, for $1 \leq i \leq d$,

$$
N_{k}^{i}=\inf \left\{h \geq 1: \xi_{N_{h}^{i-1}}^{i} \geq \limsup _{n \rightarrow \infty} \xi_{N_{n}^{i-1}}^{i}-\frac{1}{k}\right\} .
$$

Now take $N_{k}=N_{k}^{d}$.

\section{Acknowledgements}

I would like to thank Chris Rogers and an anonymous referee for reading earlier drafts of this paper and for suggesting ways to improve the presentation. I would also like to thank the students in the Advanced Financial Models course at the University of Cambridge for whom this proof was developed.

\section{References}

[1] Dalang, R. C., Morton, A. and Willinger, W. (1990). Equivalent martingale measures and no-arbitrage in stochastic securities market models. Stoch. Stoch. Reports 29, 185-201.

[2] Delbaen, F. And Schachermayer, W. (1998). The fundamental theorem of asset pricing for unbounded stochastic processes. Math. Ann. 312, 215-250.

[3] Delbaen, F. and Schachermayer, W. (2006). The Mathematics of Arbitrage. Springer, Berlin.

[4] Harrison, J. M. AND Kreps, D. M. (1979). Martingales and arbitrage in multiperiod securities markets. J. Econom. Theory 20, 381-408.

[5] Harrison, J. M. and Pliska, S. R. (1981). Martingales and stochastic integrals in the theory of continuous trading. Stoch. Process. Appl. 11, 215-260.

[6] JACKA, S. D. (1992). A martingale representation result and an application to incomplete financial markets. Math. Finance 2, 23-34.

[7] JACOD, J. AND ShiRYAev, A. N. (1998). Local martingales and the fundamental asset pricing theorems in the discrete-time case. Finance Stoch. 2, 259-273.

[8] Kabanov, Y. and Stricker, C. (2001). A teachers' note on no-arbitrage criteria. In Séminaire de Probabilités XXXV (Lecture Notes Math. 1755), Springer, Berlin, pp. 149-152.

[9] Rogers, L. C. G. (1994). Equivalent martingale measures and no-arbitrage. Stoch. Stoch. Reports 51, 41-49.

[10] Rogers, L. C. G. (1998). The origins of risk-neutral pricing and the Black-Scholes formula. In Handbook of Risk Management and Analysis, ed. C. O. Alexander, John Wiley, Chichester, pp. 81-94.

[11] Ross, S. A. (1978). A simple approach to the valuation of risky streams. J. Business 51, 453-475. 